\title{
The theoretical value of encounters with parasitized hosts for parasitoids
}

\author{
Munjong Kolss • Thomas S. Hoffmeister • Lia Hemerik
}

Received: 16 March 2006 /Revised: 20 June 2006 / Accepted: 14 July 2006 / Published online: 23 September 2006

(C) Springer-Verlag 2006

\begin{abstract}
A female parasitoid searching for hosts in a patch experiences a diminishing encounter rate with unparasitized and thus suitable hosts. To use the available time most efficiently, it constantly has to decide whether to stay in the patch and continue to search for hosts or to search for and travel to another patch in the habitat. Several informational cues can be used to optimize the searching success. Theoretically, encounters with unparasitized hosts should lead to a prolonged search in a given patch if hosts are distributed contagiously. The results of empirical studies strongly support this hypothesis. However, it has, to date, not been investigated theoretically whether encounters with already parasitized hosts (which usually entail time costs) provide a parasitoid with valuable information for the optimization of its search in depletable patches, although the empirical studies concerning this question so far have produced ambiguous results. Building on recent advances in Bayesian foraging strategies, we approached this problem by modeling a priori searching strategies (which
\end{abstract}

Communicated by H. Kokko

\section{Kolss $(\square)$}

Department of Biology, University of Fribourg,

Chemin du Musée 10,

1700 Fribourg, Switzerland

e-mail: munjong.kolss@unifr.ch

T. S. Hoffmeister

Institute of Ecology and Evolutionary Biology,

University of Bremen,

FB 02, Leobener Str. NW2,

28359 Bremen, Germany

L. Hemerik

Biometris, Department of Mathematical and Statistical Methods,

University of Wageningen,

P.O. Box 100, 6700 AC Wageningen, The Netherlands differ in the amount of information considered) and then testing them in computer simulations. By comparing the strategies, we were able to determine whether and how encounters with already parasitized hosts can yield information that can be used to enhance a parasitoid's searching success.

Keywords Parasitoid - Optimal foraging · Patch time allocation - Bayesian updating . Searching strategy $\cdot$ Stochastic model

\section{Introduction}

In nature, resources are not evenly distributed over the environment, but usually only occur in distinct patches. A foraging animal depletes the resources in a patch and thus experiences diminishing returns over time. Consequently, it constantly has to decide whether it would do better by continued searching in the currently visited patch or by leaving, searching for, and traveling to another patch in the habitat.

In the context of optimal foraging theory, much work has been conducted on this problem of patch time allocation. Especially the host searching behavior of insect parasitoids has attracted interest since parasitoids provide ideal study objects due to the direct link of their foraging success to fitness (Godfray 1994; Godfray and Shimada 1999). One central model of patch leaving decisions in depletable patches is the Marginal Value Theorem (MVT) developed by Charnov (1976), which asserts that the optimum time to leave a patch is when the instantaneous gain rate drops below the maximum average gain rate attainable in the habitat.

However, this model has been criticized (Oaten 1977; Green 1980, 1984; McNamara 1982; Stephens and Krebs 
1986; Godfray 1994) because it is based on the assumption that the forager is omniscient concerning the distribution and quality of patches and consequently does not gain any information while foraging. In nature, patches and habitats differ in quality both within and between generations, and thus, the omniscience condition obviously is not realistic. Therefore, we need to find a link between the functional optimality predictions and proximate mechanisms that can be used by foragers with limited knowledge (van Alphen and Vet 1986; Vos et al. 1998; van Alphen et al. 2003).

A simple mechanism would be the use of so-called rules of thumb: (a) leave after a fixed number of resource items have been found-fixed number rule (Gibb 1962), (b) leave after a fixed time interval-fixed time rule (Krebs 1973), and (c) leave after no resource item has been found for a fixed time interval-fixed giving-up time (GUT) rule (Hassell and May 1974; Murdoch and Oaten 1975). Waage (1979) developed a more sophisticated mechanistic model for parasitoids. He assumed that a parasitoid's responsiveness to the patch edge decreases with the time spent on the patch, e.g., through sensory habituation until a critical threshold is reached and the patch is abandoned. In this so-called increment-decay model, Waage, building on the results of his study on the searching behavior of the parasitoid Venturia (Nemeritis) canescens (Waage 1978), suggested that ovipositions increase the responsiveness and consequently lead to higher patch residence times (PRT). Contrary to the latter prediction, various studies with different parasitoids revealed that ovipositions do not always increase but can actually decrease the PRT (Strand and Vinson 1982; Casas et al. 1993; Driessen and Bernstein 1999; Wajnberg et al. 1999) or even affect the patch-leaving decision dynamically, depending on the experience and physiological state of the parasitoid (Outreman et al. 2005).

Iwasa et al. (1981) showed theoretically that increasing the PRT after host encounters is adaptive when there is large heterogeneity in patch quality, e.g., if hosts have an aggregated (overdispersed) distribution among patches since the encounter of a host then leads to an increased probability of finding another one, whereas decreasing the PRT is advantageous when hosts are more regularly distributed because in this case, the probability of finding a second host is reduced. Indeed, studies performed with parasitoids whose hosts are aggregated in space showed that ovipositions significantly increased the tendency to stay in a patch (Haccou et al. 1991; Hemerik et al. 1993).

Although the effect of ovipositions is well established both empirically and theoretically, the effect of rejections of already parasitized hosts has not yet been finally resolved. In predator-prey systems, the encountered prey items are consumed. In parasitoid-host systems, however, parasitized hosts remain in the patch and can therefore be reencountered. For at least two reasons, such encounters with already parasitized hosts may be important. First, they may entail time costs for examination and rejection. Second, encounters with hosts parasitized by a conspecific may provide the searching parasitoid with the information that the current patch has already been exploited and consequently probably contains a large fraction of parasitized hosts.

Accordingly, van Lenteren proposed (van Lenteren 1981) and found (van Lenteren 1991; but see van Alphen 1993) lower PRT and search times when parasitoids encountered parasitized hosts. However, the findings of other experiments on species whose hosts usually show a clumped distribution (in the case of regularly distributed hosts, a rejection should always be expected to increase the parasitoid's leaving tendency, as should in fact any encounter of a host) did not support this hypothesis unequivocally. No effect of rejections of already parasitized hosts on the search time was found by van Alphen and Galis (1983) for Asobara tabida; Haccou et al. (1991) detected no effect of rejection rates on the leaving tendency of Leptopilina heterotoma, whereas Varaldi et al. (2005) observed an increase in the leaving tendency after rejections for the same species. van Steenis et al. (1996) found no influence of rejections on the leaving tendency of Aphidius colemani, and Varaldi et al. (2005) detected no effect of host encounters on the leaving tendency of Leptopilina boulardi.

An unambiguous example of an effect of rejections of parasitized hosts on the PRT was provided by Hemerik et al. (1993), who confirmed an increase in the leaving tendency of experienced Leptopilina clavipes if the first host encountered in a patch was already parasitized by a conspecific. This led to the hypothesis that only the first host encounter provides relevant information: if it is with a parasitized host, the searching parasitoid gains the information that the patch has already been exploited. From the second host encounter onwards, the parasitoid, provided that it is unable to discriminate between self- and conspecifically parasitized hosts, does not know the origin of the egg in the host and therefore does not gain any further information on the extent of exploitation in the patch. However, the assumption that discrimination against self-parasitized hosts is lacking in parasitoids is not necessarily true. In fact, such discrimination has been demonstrated in several species (Visser et al. 1990; van Dijken et al. 1992; Marris et al. 1996; Wajnberg et al. 2000; Boivin et al. 2004). More recent studies (Wajnberg et al. 1999; Wajnberg et al. 2000; Boivin et al. 2004; Wang and Keller 2004) suggested that rejections of parasitized hosts may also affect the PRT at later encounters. However, in Wajnberg et al. (1999) and Wang and Keller (2004), the investigated parasitoid species, Telenomus busseolae and Diadegma semiclausum, also increased their leaving ten- 
dency after ovipositions. The same was found by Boivin et al. (2004) for Anaphes victus, whereas van Baaren et al. (2005a,b) observed a decrease in the leaving tendency after ovipositions for the same species. In Wajnberg et al. (2000), only rejections of self-parasitized (or unparasitized) hosts decreased the PRT of Trichogramma brassicae; no influence of encounters with conspecifically parasitized hosts was detected.

In view of these somewhat confusing empirical results, it is striking that theoretical investigations of this problem are almost completely lacking. Only Pierre et al. (2003) developed a model where parasitoids sample a patch to estimate the proportion of parasitized hosts. However, their model holds only for undepletable patches and does not consider travel costs. Furthermore, it is not principally concerned with the optimality of decisions.

To close this gap, we investigated for parasitoids searching for hosts in depletable patches which information they should consider to determine the optimum patch leaving strategy and how this information should be used. In particular, we were interested in the question whether parasitized hosts provide foraging parasitoids with information that should be incorporated in the decision-making process for patch leaving to maximize their long-term fitness gain rate. Since this cannot easily be solved analytically, as Pierre et al. (2003) point out, we defined a priori searching strategies (patch leaving rules) in a model. With these rules that differ in the amount of information that the parasitoids use, we ran computer simulations with a variety of different values for behavioral traits and environmental parameters to elucidate which of the different searching strategies performs best and thus what kind of information is substantial for a foraging parasitoid.

\section{The model}

Assumptions and restrictions

In each model run, only one parasitoid is currently exploiting the patches. It can, however, encounter already parasitized hosts. Consequently, direct interference is neglected, whereas indirect interference takes place. Within patches, hosts are found at random, i.e., according to a Poisson process; systematic search in a patch is disregarded. In a given patch, all present hosts have the same probability to be encountered. Upon encounter, the status of the host is determined (unparasitized, parasitized, selfmarked or not). We assume perfect host discrimination; that is, the parasitoid can infallibly distinguish between unparasitized and parasitized hosts.

Unparasitized hosts are always accepted for oviposition (and are thus turned into parasitized hosts which are not replaced, i.e., remain in the patch), and already parasitized hosts are always rejected. Therefore, no superparasitism occurs, although it has been shown that this may actually be adaptive under certain circumstances (van Alphen and Nell 1982; van Alphen and Visser 1990; van der Hoeven and Hemerik 1990; Visser et al. 1990; van Dijken et al. 1992). Perfect discrimination is a reasonable simplification to keep the model intelligible.

The distribution of hosts across patches in the habitat is assumed to follow a negative binomial distribution. Thus, given the mean number of hosts per patch $m$ and the clumping parameter $k$, the probability that a patch contains $x$ hosts is given by Eq. 1 (see, e.g., Mangel and Clark (1988) for the definition) and has mean $m$ and variance $m+\left(m^{2} / k\right)$ :

$P(x)=\left(\begin{array}{c}x+k-1 \\ x\end{array}\right)\left(\frac{k}{m+k}\right)^{k}\left(\frac{m}{m+k}\right)^{x}$

For several reasons, the negative binomial distribution is very convenient: first, the mathematical equation contains only two parameters ( $m$ and $k$ ) and can therefore be calculated easily; second, it can cover the range from random (Poisson; $k \rightarrow \infty)$ to extremely clumped $(k \rightarrow 0)$ distributions; and third, it can often adequately describe aggregated distributions of insects, as has been shown in several ecological studies (Atkinson and Shorrocks 1984; Zhang et al. 1993; Peng and Brewer 1994; Tsai et al. 2000; Hoffmeister and Rohlfs 2001).

One of the central assumptions of the model is that the foraging parasitoid maximizes its long-term rate of fitness gain, i.e., the number of host parasitizations per time unit. This is a reasonable assumption as long as the parasitoid is not egg limited (Ellers et al. 1998; Sevenster et al. 1998). Internal state constraints such as nutritional condition or limitation of egg complement and survival probability are not considered.

Decision rule

The model is based on the potential value assessment rule developed by Olsson and Holmgren (1998). These authors extended a discrete stochastic model by Iwasa et al. (1981). They showed that a randomly searching forager that updates its statistical estimate of the number of hosts in the current patch while foraging (Bayesian forager; Valone and Brown 1989; Olsson and Holmgren 1998) should not base its patch leaving decision on the instantaneous patch value (instantaneous fitness gain rate), but rather on the estimated potential patch value (potential fitness gain rate) if hosts have an aggregated distribution. Besides the estimation of the resource content of a patch, the potential patch value also includes the evaluation of the probability 
of gaining new information from which the patch can be reassessed. The rule states that the forager should leave the current patch when the potential gain rate drops below the average gain rate obtainable in the habitat (see below for details on the calculations). We extended their model by incorporating parasitized hosts.

Potential fitness gain rate

Following Iwasa et al. (1981) and Olsson and Holmgren (1998), the probability of $x$ remaining unparasitized hosts after search time $z$ in the current patch is (see Table 1 for an overview of the variables used throughout the text)

$R_{x}(z, n)=\frac{Q_{x+n}\left(\begin{array}{c}x+n \\ n\end{array}\right) e^{-a^{\prime} z x}}{\sum_{i=0}^{N_{\max }}\left[Q_{i+n}\left(\begin{array}{c}i+n \\ n\end{array}\right) e^{-a^{\prime} z i}\right]}$

where $n$ is the number of unparasitized hosts found and parasitized, $x+n$ the number of unparasitized hosts initially present in the patch, $a^{\prime}$ the searching efficiency of the forager, $N_{\max }$ the maximum number of hosts that a patch can contain (for our simulations, we set this value to 32 ), and $Q_{j}$ the probability that a patch initially contains $j$ hosts. If hosts are distributed according to a negative binomial distribution, $Q$ can be calculated from the mean and clumping parameter.

The probability to find $i$ unparasitized hosts (which are then parasitized but remain in the patch and can thus be reencountered) during the next unit of search time, given that $x$ unparasitized hosts are present before the onset of this unit of search time, follows a binomial distribution with parameters $x$ and $e^{-a^{\prime}}$ :

$$
\begin{aligned}
P_{i}(x) & =\left(\begin{array}{c}
x \\
i
\end{array}\right)\left(e^{-a^{\prime}}\right)^{x-i}\left(1-e^{-a^{\prime}}\right)^{i} \\
& =\left(\begin{array}{c}
x \\
i
\end{array}\right) e^{-a^{\prime} x}\left(e^{a^{\prime}}-1\right)^{i}
\end{aligned}
$$

With the above equations, it is possible to calculate the probability of encountering exactly $i$ unparasitized hosts in a given patch during the next unit of search time:

$S_{i}(z, n)=\sum_{x=0}^{N_{\max }-n}\left[P_{i}(x) R_{x}(z, n)\right]$

The expected number of unparasitized hosts to be found during the next unit of search time then becomes:

$S_{\text {unp. }}=\sum_{i=0}^{N_{\max }-n}\left[i S_{i}(z, n)\right]$

Given the time needed for an oviposition and the activities intrinsically connected with it (handling time $T_{\mathrm{h}}$ ), we can now calculate $F^{\prime}$, the potential fitness gain rate obtainable in the next time unit if rejections cannot occur (or the time costs of a rejection are zero) analogously to the equation for optimal diet choice (Stephens and Krebs 1986):

$F^{\prime}=\frac{S_{\text {unp. }}}{1+S_{\text {unp. }} T_{h}}$

As mentioned, this equation does not include the possibility of encounters with already parasitized hosts. To do this, the expected number of parasitized hosts that will be found during the next unit of search time $S_{\text {par. }}$ must be calculated as well. Assuming that (a) the parasitoid is able to distinguish between hosts parasitized (and marked) by itself and hosts parasitized (and exclusively marked) by a conspecific and that (b) hosts previously parasitized by a conspecific are marked as self-parasitized upon encounter (and are subsequently recognized as self-marked), the hosts parasitized by a conspecific can be considered depletable. Therefore, there are three distinct classes of hosts: (1) unparasitized hosts (depletable), (2) hosts parasitized (marked) by a conspecific (depletable), and (3) selfparasitized (self-marked) hosts (not depletable). Thus, $S_{\text {con. }}$, the expected number of conspecifically marked hosts to be found during the next unit of search time, can be calculated analogously to $S_{\text {unp. }}$. Here, the self-marked hosts are not considered yet. However, the number of self-marked hosts is known: it is the number of unparasitized hosts found $n$ plus the number of conspecifically marked hosts found $n_{\mathrm{c}}$. Thus, it is possible to calculate the expected number of already parasitized hosts $S_{\text {par. }}$ to be found during the next unit of search time as:

$S_{\text {par. }}=S_{\text {con. }}+a^{\prime}\left(n+n_{c}\right)$

Now, given the time needed for the rejection of an already parasitized host and the activities intrinsically connected with it (rejection time $T_{\mathrm{r}}$ ), it is finally possible to determine the potential fitness gain rate obtainable in the patch in the next time unit:

$F=\frac{S_{\text {unp. }}}{1+S_{\text {unp. }} T_{h}+S_{\text {par. }} T_{r}}$

Average habitat fitness gain rate

To calculate the average fitness gain rate obtainable in the habitat, the fitness gain rate obtainable in a patch of any quality has to be calculated, and the probability of the occurrence of a patch of this quality must be known. 
Table 1 Overview of the parameters used in modeling the behavioral strategies of the parasitoids

\begin{tabular}{|c|c|}
\hline Variable & Description \\
\hline$a^{\prime}$ & searching efficiency of the forager \\
\hline$F$ & potential fitness gain rate obtainable in the next time unit if rejections can occur \\
\hline$F^{\prime}$ & potential fitness gain rate obtainable in the next time unit if rejections cannot occur \\
\hline$G\left(T_{\mathrm{t}}\right)$ & fitness gain rate in the patch after total patch residence time $T_{\mathrm{t}}$ \\
\hline$G_{\max }(x, y)$ & $\begin{array}{l}\text { maximum fitness gain rate obtainable in a patch containing } x \text { unparasitized and } y \\
\text { parasitized hosts }\end{array}$ \\
\hline$H$ & average habitat fitness gain rate \\
\hline$H^{\prime}$ & average habitat fitness gain rate estimate of a parasitoid neglecting parasitized hosts \\
\hline$I(x, y)$ & probability that a patch initially contains $x$ unparasitized and $y$ parasitized hosts \\
\hline$k$ & clumping parameter of the negative binomial distribution \\
\hline$\lambda$ & mean number of host encounters per host present \\
\hline$m$ & mean of the negative binomial distribution \\
\hline$n$ & number of unparasitized hosts found and parasitized \\
\hline$n_{\mathrm{c}}$ & number of conspecifically marked hosts found (and rejected) \\
\hline$N_{\mathrm{a}}$ & expected number of unparasitized hosts encountered (after a given patch residence time) \\
\hline$N_{\mathrm{e}}$ & total number of host encounters (with unparasitized or parasitized hosts) \\
\hline$N_{\max }$ & maximum number of hosts a patch can contain \\
\hline$N_{p_{0}}$ & number of parasitized hosts initially present in the patch \\
\hline$N_{\mathrm{t}}$ & total number of hosts present in the patch \\
\hline$N_{\mathrm{u}}$ & number of currently unparasitized hosts present in the patch \\
\hline$N_{u_{0}}$ & number of unparasitized hosts initially present in the patch \\
\hline$P_{i}(x)$ & probability to find $i$ unparasitized hosts during the next unit of search time \\
\hline$Q_{j}$ & probability that a patch initially contains $j$ hosts \\
\hline$R_{x}(z, n)$ & $\begin{array}{l}\text { probability of } x \text { out of initially }(x+n) \text { unparasitized hosts remaining unparasitized after } \\
\text { search time } z \text { in the current patch }\end{array}$ \\
\hline$S_{i}(z, n)$ & $\begin{array}{l}\text { probability of encountering exactly } i \text { unparasitized hosts in the patch during the next unit } \\
\text { of search time, given that } n \text { unparasitized hosts have been found during the last } z \text { units } \\
\text { of search time }\end{array}$ \\
\hline$S_{\text {con. }}$ & expected number of conspecifically marked hosts to be found during the next unit of search time \\
\hline$S_{\text {par. }}$ & expected number of already parasitized hosts to be found during the next unit of search time \\
\hline$S_{\text {unp. }}$ & expected number of unparasitized hosts to be found during the next unit of search time \\
\hline$T_{\mathrm{h}}$ & handling time=time needed for an oviposition and the activities intrinsically connected with it \\
\hline$T_{\mathrm{r}}$ & rejection time=time needed for a host rejection and the activities intrinsically connected with it \\
\hline$T_{\mathrm{t}}$ & total patch residence time \\
\hline$T_{\text {tr }}$ & travel time between patches \\
\hline$x$ & number of unparasitized hosts in the patch \\
\hline$y$ & number of parasitized hosts in the patch \\
\hline$z$ & search time in the current patch \\
\hline
\end{tabular}

The maximum fitness gain rate obtainable in a patch of given quality can only be assessed when the theoretical number of (unparasitized) hosts found after any particular total PRT $T_{\mathrm{t}}$ is known (i.e., the expected number of ovipositions). This functional relation is the (here, stochastic) gain curve as used in the MVT, and the slope of the tangent to this gain curve - considering, of course, the average time needed to travel from one patch to the next $T_{\text {tr }}$ - equals the maximum rate of fitness gain achievable in the patch.

Here, we build upon the "attack equation" developed by Rogers (1972), which calculates the expected number of parasitized hosts as a function of total PRT $T_{\mathrm{t}}$, initial number of hosts $N_{t}$, searching efficiency $a^{\prime}$, and handling time $T_{\mathrm{h}}$ for parasitoids foraging in depletable patches. We extended this equation for parasitoid-host systems ${ }^{1}$ by including rejection time $T_{\mathrm{r}}$ and allowing parasitized hosts to be present initially. Now, if the total number of hosts in the patch $N_{\mathrm{t}}$, the number of unparasitized hosts initially present $N_{u_{0}}, T_{\mathrm{h}}, T_{\mathrm{r}}$, and $a^{\prime}$ are known, $T_{\mathrm{h}} \geq 0, T_{\mathrm{r}} \geq 0$, and only one forager is allowed in the patch, then $N_{\mathrm{a}}$, the expected

\footnotetext{
${ }^{1}$ Originally, the equation was developed for predators searching for prey; thus, the parasitoids correspond to the predators, the parasitized hosts to the consumed prey items, and $N_{\mathrm{t}}$ to the initial prey number.
} 
number of unparasitized hosts encountered, is implicitly given by (see Appendix for the derivation)

$N_{a}=N_{u_{o}}\left(1-\exp \left\{-a^{\prime}\left(T_{t}-N_{a} T_{h}-\left[\frac{N_{t}^{2}}{N_{u_{o}}} \ln \left(\frac{N_{u_{o}}}{N_{u_{o}}-N_{a}}\right)-N_{a}\right] T_{r}\right)\right\}\right)$

In the simulation program, Eq. 9 was solved numerically.

Mathematically, $N_{\mathrm{a}}$ can be expressed as a function of total PRT $T_{\mathrm{t}}$, and then the function for fitness gain rate is also a function of $T_{\mathrm{t}}$,

$G\left(T_{t}\right)=\frac{N_{a}\left(T_{t}\right)}{T_{t}+T_{t r}}$

which must be maximized, so that the maximum rate of fitness gain achievable in the patch is obtained.

For the calculation of the probabilities of the occurrence of patches with different numbers of hosts, the values given by the negative binomial distribution can be used if parasitized hosts are not initially present in a patch.

If parasitized and unparasitized hosts may initially be present in a patch (e.g., if a certain percentage of all hosts in the habitat is already parasitized), the calculation of the theoretical distribution of parasitized and unparasitized hosts across patches becomes mathematically unfeasible. As a solution, we used data obtained from preliminary simulations. For the chosen mean and clumping parameter, a habitat with 100,000 patches initially containing no parasitized hosts was set up, and a parasitoid employing strategy "A" (see below) was allowed to visit these in the following way: one patch was randomly chosen (by picking a random number from 1 to 100,000 ), and the parasitoid would search in this patch until it decided to leave; this was repeated (thus allowing any single patch to be visited several times-if the number was drawn more than once-or not at all) until the desired percentage of hosts across the total 100,000 patches was parasitized. The resulting distribution of unparasitized and parasitized hosts, as calculated from the 100,000 patches, was subsequently used in all simulations with this parameter set (i.e., for all strategies). Repeating the simulations using strategy "E" (see below) during the preliminary simulations did not have a measurable impact on the results and conclusions (data not shown).

If the probability that a patch initially contains $x$ unparasitized and $y$ parasitized hosts is $I(x, y)$ and the maximum fitness gain rate obtainable in a patch containing $x$ unparasitized and $y$ parasitized hosts is $G_{\max }(x, y)$, the average habitat fitness gain rate is given by

$H=\sum_{x=0}^{N_{\max }} \sum_{y=0}^{N_{\max }-x}\left[I(x, y) G_{\max }(x, y)\right]$

The leaving rules

As we are concerned with clumped (to Poisson) host distributions, we did not include the fixed number rule. As Iwasa et al. (1981) showed, this simple strategy will only perform well if hosts are distributed regularly. In fact, if the strategy says, "leave after zero hosts have been found," the parasitoid will spend all its time traveling between patches; if the strategy says, "leave after one host has been found" (obviously, the same holds for more than one as well), the parasitoid will get forever stuck in the first patch that contains no hosts at all. The only possibility to get this strategy to work would be to combine it with either the fixed time rule or the fixed GUT rule. This would result in an already rather complicated rule, which is difficult to implement and moreover expected not to do significantly better than the fixed (giving-up) time rule itself, if at all. Therefore, we tested the following six patch leaving strategies:

1. Fixed patch residence time (PRT) rule (" $T$ "): The parasitoid will leave the patch if it has been on the patch for a certain constant (fixed) time interval. To assure the optimum result for this leaving rule, the best time interval for each parameter set was determined by running full simulations with all possible time intervals up to a reasonable maximum (i.e., 1,000 replicate simulations with 100 patch visits for each time interval). Then the proper simulation was repeated with the obtained time interval (i.e., the one which on average yielded the highest fitness gain rate), and only this result was used in the evaluation and comparison of the performances of the different searching strategies. 
2. Fixed search time rule (" $S$ "): The parasitoid will leave the patch if it has been searching on the patch for a certain constant (fixed) time interval (PRT minus time spent on ovipositions or rejections). To assure the optimum result for this leaving rule, the best time interval for each parameter set was determined as for strategy " $T$ ".

3. Fixed giving-up time rule (" $G$ "): The parasitoid will leave the patch if it has not encountered an unparasitized host for a certain constant (fixed) time interval (giving-up time, GUT), or alternatively-in case no unparasitized host is found-if the PRT reaches or exceeds the GUT. To assure the optimum result for this leaving rule, the best time interval for each parameter set was determined as for strategy " $\mathrm{T}$ ".

4. Potential value assessment rule without consideration of parasitized hosts ("E"): The parasitoid uses the potential value assessment rule but does not consider parasitized hosts. Thus, the potential fitness gain rate in the patch is calculated according to Eq. 6, and the average habitat fitness gain rate is as follows:

$H^{\prime}=\sum_{x=0}^{N_{\max }}\left[Q_{x} G_{\max }(x, 0)\right]$

If the potential fitness gain rate in the patch drops below the average habitat fitness gain rate, the patch will be abandoned.

5. Potential value assessment rule with consideration of parasitized hosts (“A”): The parasitoid compares the potential fitness gain rate in the patch (according to Eq. 8) with the average habitat fitness gain rate (according to Eq. 11). If the former drops below the latter, the patch will be abandoned.

6. Omniscient forager ("O”): The parasitoid essentially uses the potential value assessment rule. However, it has perfect knowledge not only concerning the distri- bution of hosts in the entire habitat but also regarding the numbers of unparasitized and parasitized hosts ( $x$ and $y$, respectively) in the currently visited patch and does not need to estimate them. Hence, $S_{\text {unp. }}$ the expected number of unparasitized hosts to be found during the next unit of search time, becomes

$S_{\text {unp. }}=\sum_{i=0}^{N_{\max }-n}\left[i P_{i}(x)\right]$

and $S_{\text {par. }}$ is calculated thus,

$S_{\text {par. }}=a^{\prime} y$

Then the potential fitness gain rate is calculated according to Eq. 8 and the average habitat fitness gain rate according to Eq. 11 .

\section{Statistical analysis}

For each of the strategies and each of 108 different parameter sets (Table 2), 1,000 simulation runs (replicates) with 100 patch visits each were conducted. Note that the rejection time $T_{\mathrm{r}}$ is set equal to the handling time $T_{\mathrm{h}}$ in some cases (in sets 73 ff., Table 2). Biologically, this does not make sense since it should lead to ovipositions in both unparasitized and parasitized hosts if the parasitoid is not egg-limited (which is assumed here); therefore, $T_{\mathrm{r}}$ should in fact always be smaller than $T_{\mathrm{h}}$, as is indeed the case for most parasitoid species (L. Hemerik, personal observation). However, we included the asymptotic case to cover the entire range of possible relationships between $T_{\mathrm{h}}$ and $T_{\mathrm{r}}$, from $T_{\mathrm{r}}=0$ to $T_{\mathrm{r}}$ increasing to asymptotically equaling $T_{\mathrm{h}}$.

For each simulation run, we calculated the realized oviposition rate $(\mathrm{OR})$ as the total number of ovipositions divided by the foraging time. The influence of the used strategy

Table 2 Parameter sets for which different searching strategies were tested in computer simulations

\begin{tabular}{|c|c|c|c|c|c|c|c|}
\hline Set no. & $m$ & $k$ & $a^{\prime}$ & $T_{\text {tr }}$ & $T_{\mathrm{h}}$ & $T_{\mathrm{r}}$ & $\%$ Par \\
\hline $1-16$ & $1.5,3.0,4.5,6.0$ & 1.0 & 0.005 & 60 & 20 & 5 & $0,25,50,75$ \\
\hline $17-28$ & 3.0 & $0.3,(1.0), 3.0,10.0$ & 0.005 & 60 & 20 & 5 & $0,25,50,75$ \\
\hline $29-40$ & 3.0 & 1.0 & $0.001,(0.005), 0.010,0.020$ & 60 & 20 & 5 & $0,25,50,75$ \\
\hline $41-52$ & 3.0 & 1.0 & 0.005 & $10,(60), 180,300$ & 20 & 5 & $0,25,50,75$ \\
\hline $53-72$ & 3.0 & 1.0 & 0.005 & 60 & $1,5,20,60,120$ & 0 & $0,25,50,75$ \\
\hline $73-84$ & 3.0 & 1.0 & 0.005 & 60 & $5,(20), 60,120$ & 5 & $0,25,50,75$ \\
\hline $85-96$ & 3.0 & 1.0 & 0.005 & 60 & $20,60,120$ & 20 & $0,25,50,75$ \\
\hline $97-104$ & 3.0 & 1.0 & 0.005 & 60 & 60,120 & 60 & $0,25,50,75$ \\
\hline $105-108$ & 3.0 & 1.0 & 0.005 & 60 & 120 & 120 & $0,25,50,75$ \\
\hline
\end{tabular}

$m=$ mean number of hosts per patch, $k=$ clumping parameter of the negative binomial distribution, $a^{\prime}=$ searching efficiency, $T_{\mathrm{tr}}=$ travel time, $T_{\mathrm{h}}=$ handling time, $T_{\mathrm{r}}=$ rejection time, $\%$ Par=percentage of parasitism in the habitat. $T_{\mathrm{tr}}, T_{\mathrm{h}}$, and $T_{\mathrm{r}}$ are measured in discrete (computer) time steps ("time units"), which may be substituted by any arbitrary time interval (e.g., seconds); $a$ ' is accordingly given in number of host encounters per time unit of searching. The values in parentheses are only included to demonstrate the succession of the values of the respective parameter; they must not be considered in counting the parameter sets since they are already included in sets $1-16$. 
and the environmental $\left(m, k, T_{\text {tr }}\right.$ and the percentage of parasitism in the habitat $\% \mathrm{Par})$ and behavioral $\left(a^{\prime}, T_{\mathrm{h}}\right.$, and $\left.T_{\mathrm{r}}\right)$ parameters on the OR was analyzed with a general linear model (GLM) with the software SAS 8.02 by SAS Institute. We specified a normal distribution of the error term and identity link function. Although the residuals actually deviated slightly from a normal distribution, they were distributed symmetrically around the mean. A minimum adequate model, starting from a maximum model containing all parameters and interaction terms of the strategy with each of the other parameters, was fitted to the data. It was obtained by backward elimination of all those factors from the maximum model whose removal led to an insignificant increase in the deviance (likelihood ratio test, type 3 analysis), starting with the highest order terms as described by Crawley (1993).

\section{Results}

The mean achieved OR of the strategies are shown in Fig. 1. On average in the 108 parameter sets, the omniscient strategy ("O") clearly obtained the highest OR ( 0.00571 ovipositions per time unit). The fixed PRT strategy ("T") yielded the worst result $(0.00343 ; 60 \%$ of strategy "O"), followed by the fixed search time strategy ("S") $(0.00384 ; 67 \%)$. The performances of the remaining three strategies are between those of "O" and "S." They are all within a small range, with the potential value assessment rule considering parasitized hosts (" $\mathrm{A}$ ") being better $(0.00427 ; 75 \%)$ than either the fixed GUT rule ("G"; $0.00417 ; 73 \%$ ) or the potential value assessment rule without consideration of parasitized hosts ("E"; 0.00418 ; $73 \%)$.

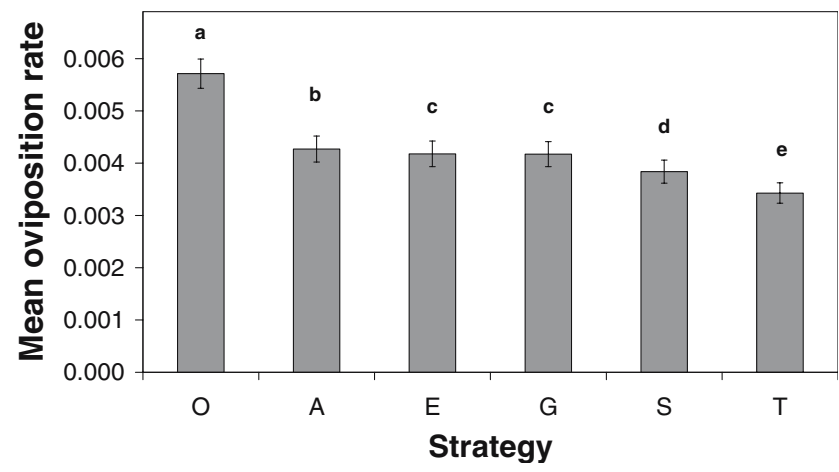

Fig. 1 Mean oviposition rates of parasitoids applying six different searching strategies (see text for details), as obtained with computer simulations. Bars with different letters were significantly different according to Tukey's studentized range test and the Ryan-EinotGabriel-Welsch multiple range test. Vertical bars represent standard errors, but note that the values were calculated from the results of simulations with 108 different parameter sets (Table 2), which leads to standard errors much higher than for any single parameter set: variation in the achieved oviposition rates was quite high among different parameter sets, but, for example, strategy "A" performed better than strategy "E" for most of these parameter sets
All main factors except for the mean number of parasitized hosts initially present in a patch (mean $N_{p_{0}}$ ) contribute significantly to the minimum adequate model (Table 3); yet, mean $N_{p_{0}}$ was not removed from the model, as it is contained in a significant interaction. Obviously, the employed strategy greatly influences the achieved OR. Post hoc tests (see legend to Fig. 1) showed that all strategies differ significantly from each other, with the one exception of the potential value assessment rule based on unparasitized hosts only ("E") and the fixed GUT rule ("G") (Fig. 1).

As expected, both the mean number of unparasitized hosts initially present in a patch (mean $N_{u_{0}}$ ) and the searching efficiency have a positive influence on the OR, whereas increasing $T_{\mathrm{tr}}, T_{\mathrm{h}}$, or $T_{\mathrm{r}}$ has a negative impact (not shown in Table 3). The effect of changing the clumping parameter $k$ of the negative binomial host distribution mainly depends on the foraging strategy; thus, the interaction of $k$ with the strategy explains more of the variance than the main factor alone (see below).

All two-factor interaction terms are significant, indicating that the effect of each of the parameters strongly depends on the strategy used by the parasitoid, i.e., that the increase or decrease in the achieved OR is greater for one strategy than for another one. For example, the strategy with fixed PRT ("T") strongly benefits relative to the others from an increase in the clumping parameter $k$, so does the strategy with fixed search time ("S"), although less so than "T." With increasing mean $N_{u_{0}}$, both potential value assessment strategies (" $\mathrm{A}$ " and "E") and the strategy with a fixed GUT ("G") gain relative to the omniscient strategy "O," whereas the fixed search time ("S")

Table 3 Factors contributing to the minimum adequate model for estimating the oviposition rate of foraging parasitoids employing different searching strategies, as calculated from data obtained with computer simulations

\begin{tabular}{lllc}
\hline Parameter & $d f$ & F value & $P$ \\
\hline Strategy & 5 & 2,563 & $<0.0001$ \\
Mean $N_{u_{0}}$ & 1 & 888,995 & $<0.0001$ \\
Mean $N_{p_{0}}$ & 1 & 3.59 & 0.058 \\
$k$ & 1 & 1,163 & $<0.0001$ \\
$a^{\prime}$ & 1 & 513,436 & $<0.0001$ \\
$T_{\text {tr }}$ & 1 & 180,939 & $<0.0001$ \\
$T_{\mathrm{h}}$ & 1 & 220,546 & $<0.0001$ \\
$T_{\mathrm{r}}$ & 1 & 21,255 & $<0.0001$ \\
Mean $N_{u_{0}} *$ Strategy & 5 & 1,180 & $<0.0001$ \\
Mean $N_{p_{0}} *$ Strategy & 5 & 41.0 & $<0.0001$ \\
$k^{*}$ Strategy & 5 & 500 & $<0.0001$ \\
$a^{\prime *}$ Strategy & 5 & 1,861 & $<0.0001$ \\
$T_{\mathrm{tr}}{ }^{*}$ Strategy & 5 & 1,560 & $<0.0001$ \\
$T_{\mathrm{h}}{ }^{*}$ Strategy & 5 & 3,418 & $<0.0001$ \\
$T_{\mathrm{r}}^{*}$ Strategy & 5 & 642 & $<0.0001$ \\
\end{tabular}

For an explanation of the variables, see Table 1. $d f=$ degrees of freedom. $R^{2}=0.85$ (thus, $85 \%$ of the total variation are explained by the model). 
and especially the fixed PRT strategy ("T") fall behind even more; the same pattern holds for the mean $N_{p_{0}}$.

The factor expected to best show the value of considering parasitized hosts, i.e., the difference between strategies " $A$ " and " $\mathrm{E}$," is the interaction of $T_{\mathrm{r}}$ with the strategy, as $T_{\mathrm{r}}$ best reflects the cost associated with the encounter of a parasitized host. Indeed, with increasing $T_{\mathrm{r}}$, the strategy with potential value assessment of unparasitized hosts only ("E") does increasingly worse when compared to the strategy with potential value assessment of both unparasitized and parasitized hosts ("A") ( $p<0.0001$; obtained by setting strategy " $\mathrm{A}$ " as the reference strategy in the GLM and thus effectively all parameter estimates of strategy "A" to zero).

Now, to proceed from the rather vague notion that parasitized hosts should be taken into consideration by the parasitoid to more concrete predictions about the expected behavioral response that can be tested experimentally, it is important to analyze how each host encounter influences the state of the potential fitness estimate. Fig. 2 shows the development of the potential fitness gain rate during fictitious patch visits for two different sequences of host encounters (left and right sides in Fig. 2) and the two strategies "A" (Fig. 2a,b) and "E" (Fig. 2c,d). Generally, the estimate declines exponen-
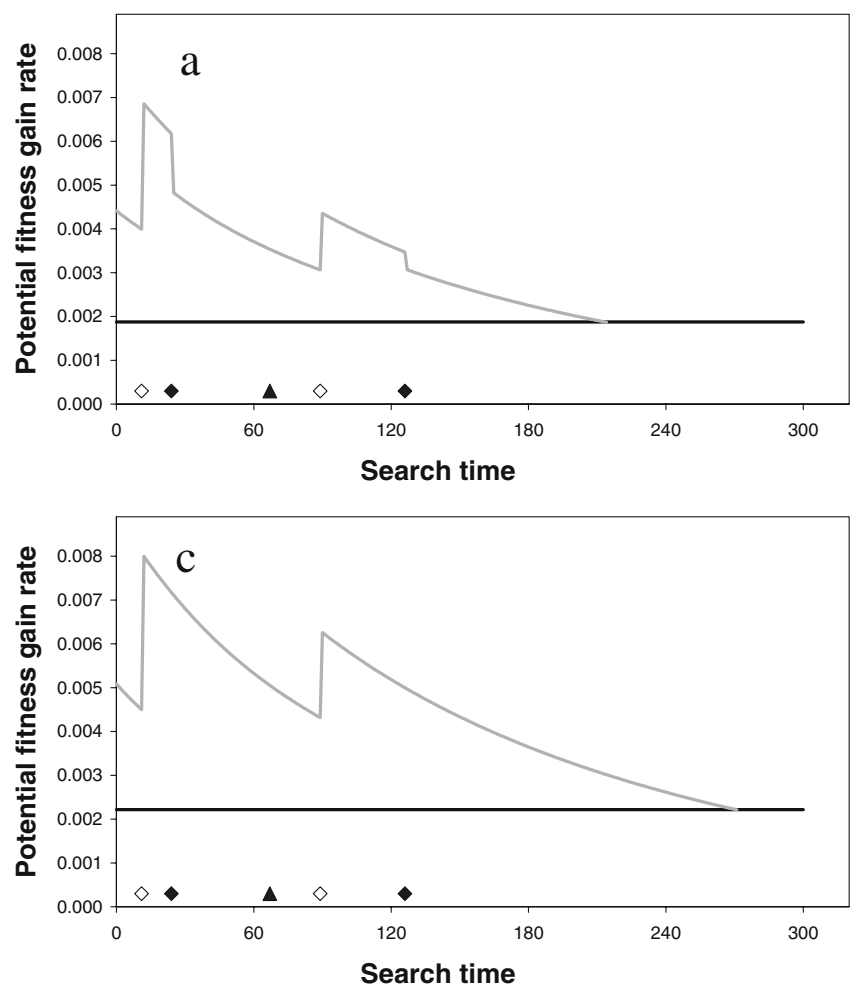

Fig. 2 Examples of the change in potential fitness gain rate (gray line) over time during patch visits for strategies "A" (a and b) and " $\mathrm{b}$ " (c and d), as obtained with computer simulations (for details on the strategies, see text). The habitat and behavioral parameters are as follows: $m=3, k=1, \% \mathrm{Par}=50 \%, a^{\prime}=0.005, T_{\mathrm{tr}}=60, T_{\mathrm{h}}=60, T_{\mathrm{r}}=30$ (see Tables 1 and 2 for explanations of these variables). The patch is abandoned when the potential fitness gain rate drops below the average habitat fitness gain rate (black line). The potential fitness gain tially over time. An oviposition leads to an increase in the estimate and thus of the search time (incremental response) The response to the rejection of a conspecifically parasitized host differs between the two strategies. Strategy "E" neglects such encounters. In contrast, " $A$ " reacts to them by decreasing its estimate of the patch value, which in turn leads to a reduction in the search time (decremental response). Note that by leaving earlier, the parasitoid employing strategy " $\mathrm{A}$ " can partially avoid the risk of losing time through potential further encounters of parasitized hosts (Fig. 2b vs d), which entail the cost of a rejection. Reencounters of self-marked hosts have no influence on the estimate of the potential fitness gain rate of both strategies and accordingly do not provide the foraging parasitoid with usable information.

\section{Discussion}

The theoretical approach

The goal of this work was to determine which informational cues a parasitoid searching for patchily distributed hosts should theoretically exploit to maximize its future searching success,
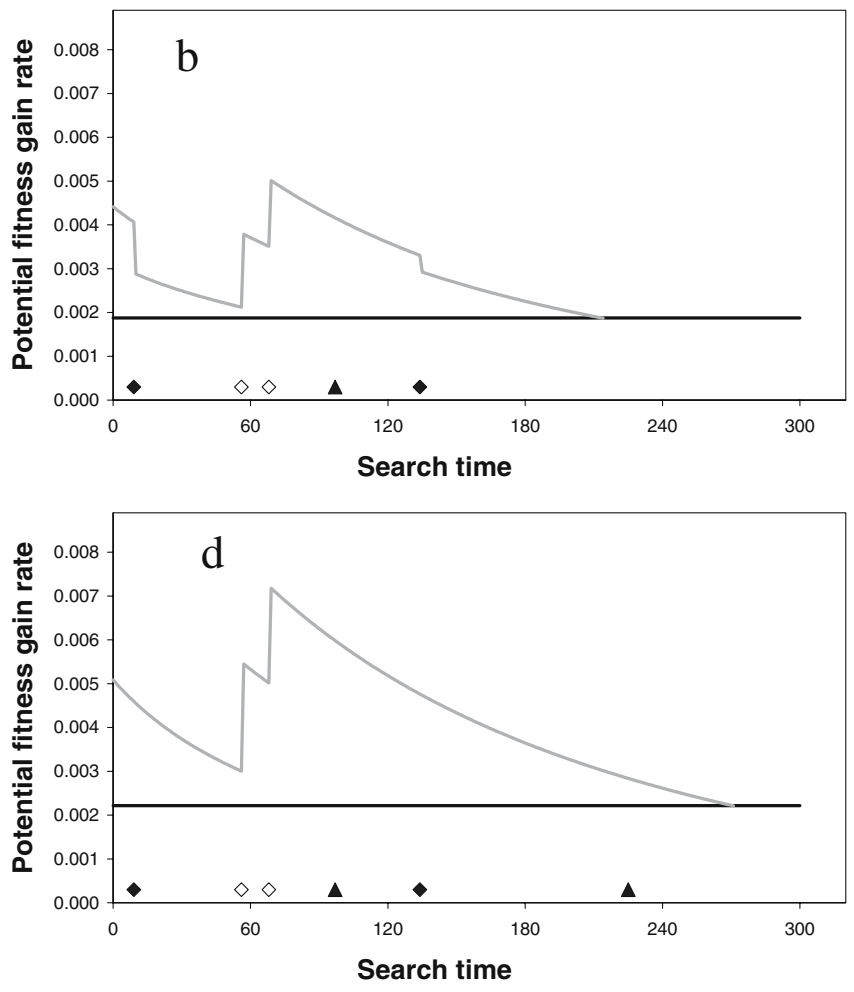

rate depends on the search time and on the experience made during the patch visit: open diamond, encounter of an unparasitized host; closed diamond, encounter of a conspecifically parasitized host; closed triangle, reencounter of a self-marked host. For better illustration of the reactions of an "A" or an "E" strategist, the sequence and timing of host encounters (which usually are stochastic events) during these sample patch visits were kept the same in panels $\mathbf{a}$ and $\mathbf{c}$ and in panels $\mathbf{b}$ and $\mathbf{d}$, respectively, by hard-coding them in the simulation program 
which is directly linked to fitness. We were not primarily concerned with the information processing mechanisms, but rather with the question whether and how a specific piece of information can be used to alter the decision-making process and thus the searching behavior in such a way that the longterm searching success is enhanced.

Here, we were especially interested in the effect of host encounters on the decision when to leave a patch. A lot of theoretical and empirical work has been performed on the influence of encounters with unparasitized hosts (see "Introduction"). However, the effect of encounters with already parasitized hosts has not been firmly established. The experimental studies conducted so far have produced ambiguous results, and until recently (Pierre et al. 2003), no one has tried to approach this question theoretically. However, the model developed by Pierre et al. (2003) is not primarily concerned with the optimality of decisions.

In the first attempt to fill this theoretical void, we modeled different searching strategies and tested them through computer simulations in different virtual habitats, i.e., environmental conditions. Naturally, any model must make simplifications and restrictions, several of which have already been discussed above, such as the assumptions of perfect host discrimination and a Poisson searching process, the exclusion of superparasitism and direct interference, and the neglect of mortality risk.

The host distribution is assumed to follow a negative binomial distribution; that is, hosts are distributed across patches in an aggregated manner. However, the modeled habitat is not spatially explicit. There is no variation in travel time during a simulation run, and all patches in the habitat have the same probability to be encountered; thus, no preference of neighboring patches or attraction to patches proportional to host density is implemented. The latter would result in relatively more frequent visits of patches with high host numbers and thus conceivably lead to a greater relative success of the more sophisticated learning strategies.

Furthermore, the average habitat quality remains constant throughout the entire simulation, which implies that the number of patches in the habitat by far exceeds the number of patches visited by the searching parasitoid (in fact, mathematically, it is assumed that the number of patches in the habitat is infinite). Thus, changes in the environment during a parasitoid's lifetime, from year to year, or from generation to generation are not considered.

Bayesian updating is restricted to the estimate of patch quality; no update of the estimate of habitat quality or host distribution is made.

Despite these limitations, the model produces reasonable results and provides an interesting tool for further research. In principle, it is possible to extend the model by incorporating several of the above aspects.
The results

Most results from the simulations do not come as a surprise. Higher mean numbers of unparasitized hosts initially present $N_{u_{0}}$ should trivially lead to higher average OR, which is confirmed by the model. Moreover, it should be expected that the assessment rules gain relative to strategy "O" when the number of unparasitized hosts and thus the frequency of encounters with such hosts increase since from more encounters, more information can be obtained. This is indeed the case, whereas the simple strategies with fixed search time ("S") and fixed PRT ("T"), which do not use the information conveyed by host encounters, lose out even more.

Higher mean numbers of parasitized hosts initially present $N_{p_{0}}$ should lead to lower OR, as long as $T_{\mathrm{r}}$ is greater than zero. This prediction is not entirely met: although a higher mean $N_{p_{0}}$ reduces the OR, this effect is not significant. It seems to be overridden by the interaction of the mean $N_{p_{0}}$ with the strategy: here again, the assessment rules [including the fixed GUT strategy ("G"), which implicitly reacts to host encounters] increase their performance relative to the omniscient strategy ("O") when the mean $N_{p_{0}}$ increases, whereas strategies with fixed search time ("S") and fixed PRT ("T") do relatively worse.

Increasing $T_{\mathrm{tr}}, T_{\mathrm{h}}$, or $T_{\mathrm{r}}$ inevitably leads to a decrease in the OR, as can be shown analytically. For our simulations, this is also confirmed by the GLM.

The clumping parameter $k$ has most of its influence in the interaction term with the strategy-its effect strongly depends on the foraging strategy used by the parasitoid. This is mainly due to strategies " $\mathrm{S}$ " and " $\mathrm{T}$ ": their $\mathrm{OR}$ greatly increases relative to the other strategies with increasing $k$ (i.e., increasing randomness in host distribution), which is in agreement with the predictions made by Iwasa et al. (1981): when the host distribution is Poisson, the estimate of the number of remaining hosts is a function of time only (i.e., host encounters do not convey any valuable information), and the potential value assessment rule resembles a fixed time (or fixed search time) strategy; it also fits with the findings of Green (1980) and Stephens (1993), according to whom the advantage of assessment is greatest when patch quality is most variable.

The rejection time parameter $T_{\mathrm{r}}$ can be expected to reveal differences between the searching strategies best; the greater it is, the more important should it be to estimate the probability of encounters with parasitized hosts (which entail time costs for rejecting the host) correctly. Indeed, for higher $T_{\mathrm{r}}$, the potential value assessment rule which considers parasitized hosts ("A") is significantly better than the one neglecting parasitized hosts ("E") $(p<0.0001)$.

Evaluation of the overall performances showed that all searching strategies differ significantly from each other except for strategies "E" and "G" (Fig. 1). The rather 
unrealistic omniscient strategy ("O") achieved the highest OR, followed by the strategy with potential value assessment based on encounters with both parasitized and unparasitized hosts ("A"; achieved OR approximately $75 \%$ compared to "O"); the fixed search time strategy ("S") and particularly the fixed PRT strategy ("T") did worst. The rather simple fixed GUT rule ("G") did quite well and was in fact virtually equal to the assessment rule which neglects parasitized hosts ("E") (both approximately $73 \%$ compared to "O").

The question whether parasitized hosts should be considered for the patch leaving decision, i.e., whether strategy " $A$ " performs better than strategy "E", can only be answered carefully. Although the difference is statistically significant, it is only about $2 \%$. However, even such a minute advantage may be important on an evolutionary scale. Whether such an advanced strategy can evolve depends among other things on proximate constraints. For example, Wajnberg et al. (2003) found in a comparative study of the Trichogrammatidae family that variation of the behavioral response to ovipositions (i.e., increasing or decreasing the PRT) was positively correlated with variation of the response to rejections of parasitized hosts. Therefore, these two features may be evolutionarily interdependent.

One of the main factors contributing to the difference between strategies " $\mathrm{A}$ " and " $\mathrm{E}$ " is the rejection time. As rejection times of real parasitoids are generally relatively short, it will presumably be difficult to explore experimentally whether a certain species considers or neglects parasitized hosts. In addition, some of the assumptions made in the current model may prove to be questionable: it is well conceivable that parasitoids (or foragers in general) are capable of updating their estimate of the average habitat quality on the basis of experience, which should be advantageous when temporal variability or superpatches are considered (Thiel and Hoffmeister 2004; Outreman et al. 2005).

Bearing in mind that the presented model quickly becomes mathematically intractable when these modifications are to be incorporated, one may wonder how a real forager in the field is supposed to be able to make all those calculations. However, as McNamara (1982) points out, an animal does not solve a decision problem by mathematics but employs a mechanism that implements a policy; no calculations are done in the mathematical sense.

Looking for a proximate mechanism that ensures optimal behavior as derived from the model, there is a striking similarity between the development of the potential value in the course of a patch visit (Fig. 2) and the simple model formulated by Waage (1979). In fact, there are only few minor differences: (1) the decay (in the potential value) over time is negative exponential rather than linear [in fact, this follows directly from the model developed by Iwasa et al. (1981); also, the models derived by McNamara (1982) and Green (1984), using quite different approaches, show very similar decay patterns]; (2) the value of the increment (of the patch value estimate) after ovipositions is time dependent, although the effect on the search time is constant; (3) the effect of encounters with parasitized hosts depends on the strategy and on whether or not the host was self-marked (i.e., known before).

An update in the estimate of the average habitat quality can easily be made by altering the leaving threshold value accordingly, with a high estimate of the average habitat quality resulting in a high leaving threshold (and thus earlier leaving); alternatively, it could be achieved by adjusting the initial level of responsiveness (Shettleworth 1998), with a high estimate of the average habitat quality corresponding to a low initial level of responsiveness and vice versa. In the mathematical model, such an update is not as simple: for example, a change in the estimate of the mean host number also implies changes in the distributions of unparasitized and parasitized hosts, which are needed in the calculation of the potential value.

It is possible to analyze the behavior of an animal acting according to a mechanism as outlined above via Cox regression (Cox 1972, 1975; Haccou and Hemerik 1985; Kalbfleisch and Prentice 2002). Indeed, studies performed so far (Hemerik et al. 1993) indicate that such a complicated network of interconnected influencing factors is involved in the patch leaving decision-making process of foraging parasitoids.

Interestingly, not all parasitoid species studied so far exhibit behavior according to the predictions of our model. Although results for L. clavipes (Hemerik et al. 1993) and L. heterotoma (Varaldi et al. 2005; Hoffmeister et al., unpublished) are in good agreement with the model predictions, T. brassicae deviates in an important detail (Wajnberg et al. 2000): here, rejections of self-parasitized hosts led to a decrease in the PRT, whereas rejections of conspecifically parasitized hosts had no effect, which is directly the opposite of what would be expected from the model. Whether this is an exceptional case remains to be resolved in further experiments. With our model, we provide a theoretical framework that yields testable predictions for future comparative studies.

\section{Conclusions}

Animal behavior is highly complex. Foraging parasitoids are known to include information gained before (Roitberg et al. 1992; Visser et al. 1992; Hoffmeister et al. 2000), during (Driessen and Bernstein 1999 and references therein; Pierre et al. 2003; van Alphen et al. 2003), and between (Thiel and Hoffmeister 2004; Outreman et al. 2005) patch visits in their decision-making process. However, we are far from understanding all aspects of this process. In the case of information gained from encounters with already parasitized hosts, even the question of its functional value was unresolved until now. 
To embark upon this problem, we developed the model presented in this work. The results of computer simulations suggest that parasitized hosts should be considered for the decision when to leave a patch, although the potential gain by doing so is relatively small. Decreasing the PRT may be adaptive when encountering a conspecifically parasitized host; reencounters of parasitized hosts should have no effect on the search time.

Despite the drawbacks inherent to any model (restrictions, assumptions, and simplification of reality), the new model provides an interesting and valuable tool for further investigation: it yields results that are in agreement with empirical studies and thus helps partially understand observed behavior from a theoretical (optimality) perspective, and it helps make testable predictions on the behavior of foragers under certain environmental conditions in the laboratory and in nature.

Acknowledgments This work is based on a thesis conducted at the laboratory of T. S. Hoffmeister at Kiel University. We thank Eric Wajnberg and two anonymous reviewers for many helpful comments and suggestions on earlier versions of this manuscript.

\section{Appendix}

The total number of hosts in a patch (at all times) $N_{\mathrm{t}}$ can be described as (see Table 1 for an explanation of the variables):

$N_{t}=N_{p_{0}}+N_{u_{0}}$

where $N_{p_{0}}$ and $N_{u_{0}}$ are the numbers of parasitized and unparasitized hosts, respectively, initially present in the patch. The mean number of host encounters per host present $\lambda$ can be defined as

$\lambda=N_{e} / N_{t}$

where $N_{\mathrm{e}}$ is the total number of host encounters (with unparasitized or parasitized hosts) in the patch.

Assuming a Poisson process for host encounters (random search, equal probability for unparasitized and parasitized hosts to be found), the probability to encounter $i$ hosts in the patch is

$P($ i encounters $)=\frac{\lambda^{i}}{i !} e^{-\lambda}$

The probability that zero parasitizations (=encounters with unparasitized hosts $=$ ovipositions) have occurred conditional on $N_{\mathrm{e}}$ is

$P\left(0\right.$ parasitizations $\mid N_{e}$ encounters $)=\left(\frac{N_{p_{0}}}{N_{t}}\right)^{N_{e}}$
Therefore, the probability of encountering $N_{\mathrm{e}}$ hosts and having zero parasitizations is

$P\left(0\right.$ parasitizations $\wedge N_{e}$ encounters $)$

$$
=\left(\frac{N_{p_{0}}}{N_{t}}\right)^{N_{e}} \frac{\lambda^{N_{e}}}{N_{e} !} e^{-\lambda}
$$

Now, irrespective of $N_{\mathrm{e}}$, the expected probability of encountering zero of the initially present $N_{u_{0}}$ unparasitized hosts and therefore having zero parasitizations is

$$
\begin{aligned}
\sum_{i=0}^{\infty}\left[\left(\frac{N_{p_{0}}}{N_{t}}\right)^{i} \frac{\lambda^{i}}{i !} e^{-\lambda}\right] & =e^{-\lambda} \sum_{i=0}^{\infty}\left[\left(\frac{N_{p_{0}}}{N_{t}} \lambda\right)^{i} \frac{1}{i !}\right] \\
& =e^{-\lambda} e^{\frac{N_{p_{0}}}{N_{t}} \lambda}=e^{-\lambda\left(\frac{N_{u_{0}}}{N_{t}}\right)}
\end{aligned}
$$

Thus, the expected number of currently unparasitized hosts $N_{\mathrm{u}}$ is:

$N_{u}=N_{u_{0}} e^{-\lambda \frac{N_{u_{0}}}{N_{t}}}$

Because of the obvious relation $N_{u}=N_{u_{0}}-N_{a}$, with $N_{\mathrm{a}}$ the number of unparasitized hosts encountered, it is possible to calculate $\lambda$ without $N_{\mathrm{e}}$, thus eliminating one of the unknown variables. $N_{u}-N_{a}=N_{u_{0}} e^{-\lambda \frac{N_{u_{0}}}{N_{t}}}$ will give us

$\lambda=\frac{N_{t}}{N_{u_{0}}} \ln \left(\frac{N_{u_{0}}}{N_{u_{0}}-N_{a}}\right)$

If only one parasitoid is allowed in the patch (number of foraging parasitoids $P_{\mathrm{t}}=1$ ), the "attack equation" developed by Rogers (1972) for foragers in depletable patches, by substituting the respective terms, can now be modified as follows ( $z=$ search time):

$$
\begin{aligned}
N_{a}= & N_{u_{0}}\left(1-\exp \left\{-a^{\prime} z\right\}\right) \\
= & N_{u_{0}}\left(1-\exp \left\{-a^{\prime}\left(T_{t}-N_{a} T_{h}-N_{r} T_{r}\right)\right\}\right) \\
= & N_{u_{0}}\left(1-\exp \left\{-a^{\prime}\left(T_{t}-N_{a} T_{h}-\left[N_{e}-N_{a}\right] T_{r}\right)\right\}\right) \\
= & N_{u_{0}}\left(1-\exp \left\{-a^{\prime}\left(T_{t}-N_{a} T_{h}-\left[\lambda N_{t}-N_{a}\right] T_{r}\right)\right\}\right) \\
= & N_{u_{0}}\left(1-\exp \left\{-a^{\prime}\left(T_{t}-N_{a} T_{h}\right.\right.\right. \\
& \left.\left.\left.\quad-\left[\frac{N_{t}^{2}}{N_{u_{0}}} \ln \left(\frac{N_{u_{0}}}{N_{u_{0}}-N_{a}}\right)-N_{a}\right] T_{r}\right)\right\}\right)
\end{aligned}
$$

\section{References}

Atkinson WD, Shorrocks B (1984) Aggregation of larval Diptera over discrete and ephemeral breeding sites: the implications for coexistence. Am Nat 124:336-351

Boivin G, Fauvergue X, Wajnberg E (2004) Optimal patch residence time in egg parasitoids: innate versus learned estimate of patch quality. Oecologia 138:640-647 
Casas J, Gurney WSC, Nisbet R, Roux O (1993) A probabilistic model for the functional response of a parasitoid at the behavioural time-scale. J Anim Ecol 62:194-204

Charnov EL (1976) Optimal foraging, the marginal value theorem. Theor Popul Biol 9:129-136

Cox DR (1972) Regression models and life tables. J R Stat Soc Ser B $34: 187-220$

Cox DR (1975) Partial likelihood. Biometrika 62:269-276

Crawley MJ (1993) GLIM for ecologists. Blackwell Scientific Publications, Oxford

Driessen G, Bernstein C (1999) Patch departure mechanisms and optimal host exploitation in an insect parasitoid. J Anim Ecol 68:445-459

Ellers J, van Alphen JJM, Sevenster JG (1998) A field study of sizefitness relationships in the parasitoid Asobara tabida. J Anim Ecol 67:318-324

Gibb JA (1962) Tinbergen's hypothesis of the role of specific search images. Ibis 104:106-111

Godfray HCJ (1994) Parasitoids: behavioral and evolutionary ecology. Princeton University Press, Princeton

Godfray HCJ, Shimada M (1999) Parasitoids as model organisms for ecologists. Res Popul Ecol 41:3-10

Green RF (1980) Bayesian birds: a simple example of Oaten's stochastic model of optimal foraging. Theor Popul Biol 18:244 256

Green RF (1984) Stopping rules for optimal foragers. Am Nat 123:30-43

Haccou P, Hemerik L (1985) The influence of larval dispersal in the cinnabar moth (Tyria jacobaeae) on predation by the red wood ant (Formica polyctena): an analysis based on the proportional hazards model. J Anim Ecol 54:755-769

Haccou P, de Vlas SJ, van Alphen JJM, Visser ME (1991) Information processing by foragers: effects of intra-patch experience on the leaving tendency of Leptopilina heterotoma. J Anim Ecol 60:93-106

Hassell MP, May RM (1974) Aggregation of predators and insect parasites and its effect on stability. J Anim Ecol 43:567-594

Hemerik L, Driessen G, Haccou P (1993) Effects of intra-patch experiences on patch time, search time and searching efficiency of the parasitoid Leptopilina clavipes. J Anim Ecol 62:33-44

Hoffmeister TS, Rohlfs M (2001) Aggregative egg distributions may promote species co-existence - but why do they exist? Evol Ecol Res 3:37-50

Hoffmeister TS, Thiel A, Kock B, Babendreier D, Kuhlmann U (2000) Pre-patch experience affects the egg distribution pattern in a polyembryonic parasitoid of moth egg batches. Ethology 106:145-157

Iwasa Y, Higashi M, Yamamura N (1981) Prey distribution as a factor determining the choice of optimal foraging strategy. Am Nat 117:710-723

Kalbfleisch JD, Prentice RL (2002) The statistical analysis of failure time data, 2 edn. Wiley, New York

Krebs JR (1973) Behavioral aspects of predation. In: Bateson PPG, Klopfer PH (eds) Perspectives in ethology. Plenum, New York, pp 73-111

Mangel M, Clark CW (1988) Dynamic modeling in behavioral ecology. Princeton University Press, Princeton

Marris GC, Hubbard SF, Scrimgeour C (1996) The perception of genetic similarity by the solitary parthenogenetic parasitoid Venturia canescens, and its effects on the occurrence of superparasitism. Entomol Exp Appl 78:167-174

McNamara J (1982) Optimal patch use in a stochastic environment. Theor Popul Biol 21:269-288

Murdoch WW, Oaten A (1975) Predation and population stability. Adv Ecol Res 9:1-131

Oaten A (1977) Optimal foraging in patches: a case for stochasticity. Theor Popul Biol 12:263-285
Olsson O, Holmgren NMA (1998) The survival-rate-maximizing policy for Bayesian foragers: wait for good news. Behav Ecol 9:345-353

Outreman Y, Le Ralec A, Wajnberg E, Pierre JS (2005) Effects of within- and among-patch experiences on the patch-leaving decision rules in an insect parasitoid. Behav Ecol Sociobiol 58:208-217

Peng CW, Brewer GJ (1994) Spatial distribution of the red sunflower seed weevil (Coleoptera: Curculionidae) on sunflower. Environ Entomol 23:1101-1105

Pierre JS, Baaren Jv, Boivin G (2003) Patch leaving decision rules in parasitoids: do they use sequential decisional sampling? Behav Ecol Sociobiol 54:147-155

Rogers D (1972) Random search and insect population models. J Anim Ecol 41:369-383

Roitberg BD, Mangel M, Lalonde RG, Roitberg CA, van Alphen JJM, Vet LEM (1992) Seasonal dynamic shifts in patch exploitation by parasitic wasps. Behav Ecol 3:156-165

Sevenster JG, Ellers J, Driessen G (1998) An evolutionary argument for time limitation. Evolution 52:1241-1244

Shettleworth SJ (1998) Cognition, evolution, and behavior. Oxford University Press, New York

Stephens DW (1993) Learning and behavioral ecology: incomplete information and environmental predictability. In: Papaj DR, Lewis AC (eds) Insect learning: ecological and evolutionary perspectives. Chapman \& Hall, New York, pp 195-218

Stephens DW, Krebs JR (1986) Foraging theory. Princeton University Press, Princeton

Strand MR, Vinson SB (1982) Behavioral response of the parasitoid Cardiochiles nigriceps to a kairomone. Entomol Exp Appl 31:308-315

Thiel A, Hoffmeister TS (2004) Knowing your habitat: linking patchencounter rate and patch exploitation in parasitoids. Behav Ecol $15: 419-425$

Tsai JH, Wang J-J, Liu Y-H (2000) Sampling of Diaphorina citri (Homoptera: Psyllidae) on orange jessamine in southern Florida. Fla Entomol 83:446-459

Valone TJ, Brown JS (1989) Measuring patch assessment abilities of desert granivores. Ecology 70:1800-1810

van Alphen JJM (1993) Patch residence time and encounters with parasitised hosts: a reaction. Neth J Zool 43:340-349

van Alphen JJM, Nell HW (1982) Superparasitism and host discrimination by Asobara tabida Nees (Braconidae, Alysiinae), a larval parasitoid of Drosophilidae. Neth J Zool 32:232-260

van Alphen JJM, Galis F (1983) Patch time allocation and parasitization efficiency of Asobara tabida, a larval parasitoid of Drosophila. J Anim Ecol 52:937-952

van Alphen JJM, Vet LEM (1986) An evolutionary approach to host finding and selection. In: Waage JK, Greathead DJ (eds) Insect parasitoids. Academic, London, pp 23-61

van Alphen JJM, Visser ME (1990) Superparasitism as an adaptive strategy for insect parasitoids. Annu Rev Entomol 35:59-79

van Alphen JJM, Bernstein C, Driessen G (2003) Information acquisition and time allocation in insect parasitoids. Trends Ecol Evol 18:81-87

van Baaren J, Boivin G, Outreman Y (2005a) Patch exploitation strategy by an egg parasitoid in constant or variable environment. Ecol Entomol 30:502-509

van Baaren J, Outreman Y, Boivin G (2005b) Effect of low temperature exposure on oviposition behaviour and patch exploitation strategy in parasitic wasps. Anim Behav 70:153-163

van der Hoeven N, Hemerik L (1990) Superparasitism as an ESS - to reject or not to reject, that is the question. J Theor Biol 146:467-482

van Dijken MJ, van Stratum P, van Alphen JJM (1992) Recognition of individual-specific marked parasitized hosts by the solitary parasitoid Epidinocarsis lopezi. Behav Ecol Sociobiol 30:77-82 
van Lenteren JC (1981) Host discrimination by parasitoids. In Nordlund DA, Jones RL, Lewis WJ (eds) Semiochemicals: their role in pest control. Wiley, New York, pp 153-179

van Lenteren JC (1991) Encounters with parasitized hosts: to leave or not to leave a patch. Neth J Zool 41:144-157

van Steenis MJ, El-Khawass KAMH, Hemerik L, van Lenteren JC (1996) Time allocation of the parasitoid Aphidius colemani (Hymenoptera: Aphidiidae) foraging for Aphis gossypii (Homoptera: Aphidae) on cucumber leaves. J Insect Behav 9:283-295

Varaldi J, Fouillet P, Boulétreau M, Fleury F (2005) Superparasitism acceptance and patch-leaving mechanisms in parasitoids: a comparison between two sympatric wasps. Anim Behav 69:1227-1234

Visser ME, van Alphen JJM, Nell HW (1990) Adaptive superparasitism and patch time allocation in solitary parasitoids: the influence of the number of parasitoids depleting a patch. Behaviour 114:21-36

Visser ME, van Alphen JJM, Nell HW (1992) Adaptive superparasitism and patch time allocation in solitary parasitoids: the influence of pre-patch experience. Behav Ecol Sociobiol 31:163-171

Vos M, Hemerik L, Vet LEM (1998) Patch exploitation by the parasitoids Cotesia rubecula and Cotesia glomerata in multi-patch environments with different host distributions. J Anim Ecol 67:774-783
Waage JK (1978) Arrestment responses of the parasitoid, Nemeritis canescens, to a contact chemical produced by its host, Plodia interpunctella. Physiol Entomol 3:135-146

Waage JK (1979) Foraging for patchily-distributed hosts by the parasitoid, Nemeritis canescens. J Anim Ecol 48:353-371

Wajnberg E, Rosi MC, Colazza S (1999) Genetic variation in patch time allocation in a parasitic wasp. J Anim Ecol 68:121-133

Wajnberg E, Fauvergue X, Pons O (2000) Patch leaving decision rules and the Marginal Value Theorem: an experimental analysis and a simulation model. Behav Ecol 11:577-586

Wajnberg E, Gonsard PA, Tabone E, Curty C, Lezcano N, Colazza S (2003) A comparative analysis of patch-leaving decision rules in a parasitoid family. J Anim Ecol 72:618-626

Wang XG, Keller MA (2004) Patch time allocation by the parasitoid Diadegma semiclausum (Hymenoptera: Ichneumonidae). III. Effects of kairomone sources and previous parasitism. J Insect Behav 17:761-776

Zhang Z-Q, Chen P-R, Wang K, Wang X-Y (1993) Overdispersion of Allothrombium pulvinum larvae (Acari: Trombidiidae) parasitic on Aphis gossypii (Homoptera: Aphididae) in cotton fields. Ecol Entomol 18:379-384 